\title{
Seam Carving for Text Line Extraction on Color and Grayscale Historical Manuscripts
}

\author{
Nikolaos Arvanitopoulos and Sabine Süsstrunk \\ School of Computer and Communication Sciences (IC) \\ École Polytechnique Fédérale de Lausanne (EPFL), Switzerland \\ nick.arvanitopoulos,sabine.susstrunk@epfl.ch
}

\begin{abstract}
We propose a novel algorithm for automatic text line extraction on color and grayscale manuscript pages without prior binarization. Our algorithm is based on seam carving to compute separating seams between text lines. Seam carving is likely to produce seams that move through gaps between neighboring lines, if no information about the text geometry is incorporated into the problem. By constraining the optimization procedure inside the region between two consecutive text lines, we can produce robust separating seams that do not cut through word and line components. Extensive experimental evaluations on diverse manuscript pages show that we improve upon the state-of-the-art for grayscale text line extraction.
\end{abstract}

\section{INTRODUCTION}

An important step in the handwriting recognition process is that of text line extraction: it aims at extracting individual text lines from the text regions of the manuscript page. It is an essential preprocessing step for many applications, such as word spotting, keyword searching, and script alignment and recognition. We propose a binarization-free text line extraction method based on seam carving, an algorithm that has been applied to image resizing [1]. Our goal is to compute separating seams between two consecutive text lines without cutting through line components. Seam carving is a suitable algorithm for this application, because it computes minimum energy seams in an image. In our problem, high-energy regions correspond to text components and low-energy regions correspond to paper or parchment background.

However, unconstrained seam carving does not take into account any prior knowledge about the text layout of the manuscript page. Therefore, the computed seams are likely to pass through gaps between multiple text lines, if these are the lowest energy regions of the neighboring image space. By constraining the seam computation between two consecutive text lines, we are able to generate a separating seam that does not assign text parts to wrong lines. To address this problem, we use a modified version of the projection profile matching

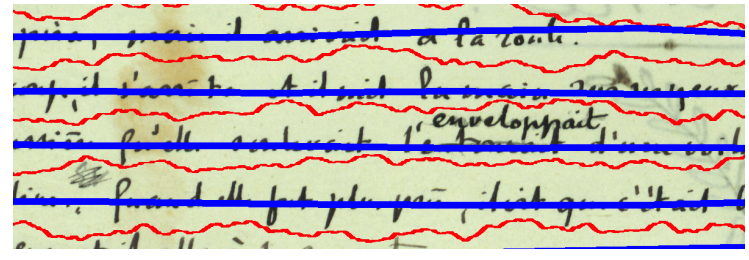

Fig. 1. Examples of computed medial seams (blue) and separating seams (red) on an extract of the work Aline of C.F. Ramuz.

approach of [2]. This method creates medial seams that can successfully approximate the orientation of each text line (see Fig. 1).

An important property of our method is that it can be directly applied to a grayscale manuscript page without any prior binarization. The generated seams can be overlaid on the original color page, as shown in Fig. 1 . This property gives a major advantage to our method, because even the most robust algorithm can produce unreliable results when applied to a binary image. The reason is that, depending on the quality of the manuscript page, the information loss introduced by the binarization procedure can be substantial. An example is shown in Fig. 2, where we apply our algorithm to the original grayscale manuscript and its binary version computed with the adaptive algorithm of [3]. Due to the low quality of the manuscript, the binarization method results in extensive loss of information, thus rendering nonapplicable any algorithm based on a binary input.

Our algorithm is general and can be applied to manuscripts of different languages, handwritings and time periods. We conduct experiments on the pages of the work Aline of the important Swiss-French writer Charles-Ferdinand Ramuz (see Fig. 3 for an extract of a page). We are able to obtain very high text line separation accuracy in this challenging collection. Additional experimental evaluation conducted on the diverse dataset of [4] shows that we can obtain state-of-the-art results for color and grayscale text line extraction. 


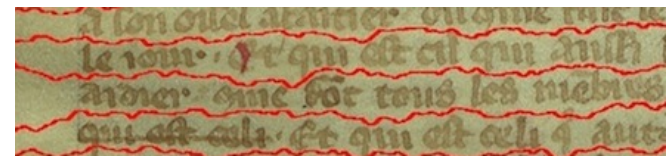

(a) Seam results using color input.

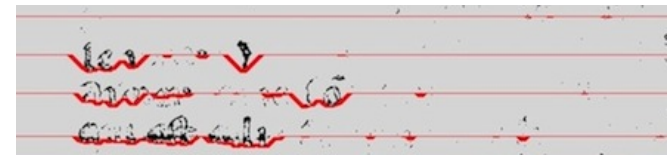

(b) Seam results using binary input.

Fig. 2. A comparison of our algorithm on color and binary input. The extensive information loss renders our algorithm unreliable for separating seam computation.

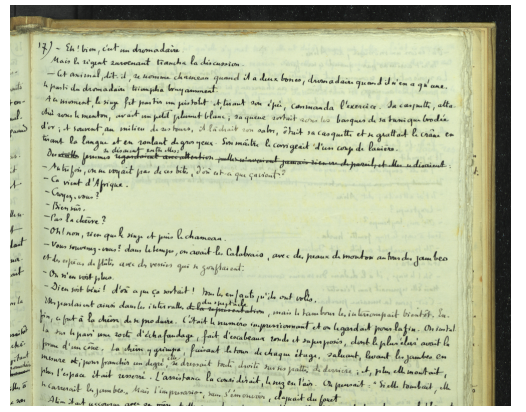

Fig. 3. Extract from a page of Aline, p. 46, C.F. Ramuz.

\section{RELATED WORK}

Most of the state-of-the-art text line extraction approaches operate on a binary image of the historical manuscript, because the location of the text is known and the extraction process becomes more efficient. The works of [5], [6] are based on horizontal projection profile analysis, with additional post-processing steps based on properties of the text connected components. The approaches proposed in [7], [8] are based on the Hough transform, which detects straight lines in images. Smearing methods are proposed in [9], [10], [11], where the goal is to group together homogeneous blocks of the manuscript page. One method, based on dynamic programming, computes separating seams with minimum cost between two consecutive lines [2] and has been extensively used in automatic transcription and ground truth creation of historical manuscripts [12], [13]. Other methods address the problem of multi-orientation by using anisotropic filters and active contours over detected ridges [14], [15]. A similar approach is proposed in [16], where local minima tracers are used to split an image into text lines. A general method for multi-oriented text line extraction on Arabic documents based on image meshing is proposed in [17].
Lately, four approaches were proposed that do not necessitate binarization and work directly on color manuscript pages. In [18], text lines are extracted based on feature classification from interest points of the original manuscript image. The authors in [19] extend the above algorithm to handle curved text lines. A hybrid approach [20] extracts text lines from layout analysis results and refines them with the help of the binary version of the manuscript. The above three approaches are proposed within the HisDoc project [21].

Our method is closely related to the fourth method proposed in [4], where the authors use a two-stage procedure to extract text lines from a grayscale image. First, seam carving is used to generate the medial seams of the manuscript page. The input to the optimization procedure is the grayscale geodesic distance transform, in which each pixel's value is its shortest path length to the nearest background pixel [22]. In a second step, seam seeds are generated and a greedy algorithm is applied, which propagates these seeds to generate two separating seams: one above and one below the medial seam. These separating seams define the upper and lower boundaries of the text line. Our algorithm differs from the above approach in the sense that we apply seam carving to directly compute the separating seams and not the medial ones. This results in a clearer separation of text lines with no cuts through letter components, which is a common phenomenon with the greedy approach of [4]. Furthermore, the use of seam carving for medial seam computation as in [4] can result in seams that jump over neighboring lines, especially in cases where the gaps between words are large compared to the distance between two consecutive text lines. Our histogram matching approach, however, is more robust, as it avoids jumping over neighboring lines while following the multiple orientation of the text.

\section{OUR APPROACH}

Our proposed method consists of two stages:

1) Medial seam computation using a projection profile matching approach similar to [2].

2) Separating seam computation using a modification of the seam carving procedure [1].

In the following two sections we describe these two stages in detail. We use the convention that an image $\mathbf{I} \in \mathbb{R}^{n \times m}$ converted to grayscale has $n$ rows and $m$ columns. The notation $I_{i, j}$ denotes the image value at the $i$-th row and $j$-th column. The coordinate system has its origin at the upper left corner of the image. 


\section{A. Medial Seam Computation}

Our medial seam computation method is inspired by the projection profile matching approach of [2]. We split the page vertically into $r$ slices, each one of width $w=\lfloor m / r\rfloor$. We apply the Sobel operator to $\mathbf{I}$ to compute its edge image $\mathbf{S} \in \mathbb{R}^{n \times m}$. We calculate smoothed horizontal projection profiles $\mathbf{P}_{g}^{c}$ of $\mathbf{S}$ in each slice independently:

$$
\begin{aligned}
& P_{i}^{c}=\sum_{j=k}^{k+w-1} S_{i, j}, \quad \mathbf{P}^{c}=\left\{P_{i}^{c}\right\}_{i=1}^{n}, \quad \mathbf{P}_{g}^{c}=g\left(\mathbf{P}^{c}\right), \\
& c=1, \ldots, r, \quad k \in\{1,1+w, \ldots, 1+(r-1) w\},
\end{aligned}
$$

where $g$ is a cubic spline smoothing filter. We denote the local maxima locations of the $c$-th profile by $L_{h}^{c}, h=$ $1, \ldots, l$ and those of $(c+1)$-th by $L_{h^{\prime}}^{c+1}, h^{\prime}=1, \ldots, l^{\prime}$. Here, $l$ and $l^{\prime}$ denote the, potentially different, number of maxima found at profiles $c$ and $c+1$ respectively. For each maximum location of profile $c$, we find the closest maximum location of profile $c+1$ and for each maximum location of profile $c+1$, we find the closest maximum location of profile $c$ :

$$
\begin{aligned}
& \operatorname{match}\left(L_{h}^{c}\right)=\arg \min _{L_{h^{\prime}}^{c+1}}\left|L_{h}^{c}-L_{h^{\prime}}^{c+1}\right|, h=1, \ldots, l, \\
& \operatorname{match}\left(L_{h^{\prime}}^{c+1}\right)=\arg \min _{L_{h}^{c}}\left|L_{h^{\prime}}^{c+1}-L_{h}^{c}\right|, h^{\prime}=1, \ldots, l^{\prime} .
\end{aligned}
$$

If the above matched locations in (2) and (3) agree, they are connected with a line. The above procedure is repeated until all slices are processed. The text line locations can now be represented in matrix form $L_{h, j}, \quad h=1, \ldots, l, j=1, \ldots, m$, where each element $L_{h, j}$ contains the $i$-th coordinate of the $h$-th line, and $l$ is the final number of lines found.

The proposed method creates piece-wise linear seams that approximate the medial axis of the text lines in the manuscript page. Any two consecutive seams define a region in which the seam carving computation is constrained. This constraint enforces the separating seam to pass between two consecutive text lines, and thus, it prevents it from assigning text parts to wrong lines.

\section{B. Separating Seam Computation}

We adapt the seam carving algorithm proposed in [1] to compute the separating seams. We include the regional constraints of the computed medial seams and modify the seam computation so that it can handle non-rectangular image regions. The energy map is the derivative image of the grayscale manuscript page:

$$
E_{i, j}=\left|\frac{I_{i, j+1}^{\sigma}-I_{i, j-1}^{\sigma}}{2}\right|+\left|\frac{I_{i+1, j}^{\sigma}-I_{i-1, j}^{\sigma}}{2}\right|,
$$

where $\mathbf{I}^{\sigma}$ is the original grayscale image smoothed with a Gaussian filter of standard deviation $\sigma$. On this map, high-energy regions correspond to text components and low-energy regions correspond to parchment background.

Let us denote the energy map between two text lines by $\mathbf{E}_{h}=\mathbf{E}_{\mathbf{J}}$, where $\mathbf{J}$ is a two-dimensional grid of width $m$, where the $j$-th column contains all the intermediate $i$ coordinates between two text line locations, that is, $\mathbf{J}_{j}=$ $\left\{L_{h, j}, \ldots, L_{h+1, j}\right\}^{T}, h=1, \ldots, l-1, j=1, \ldots, m$. A seam that passes horizontally through an image grid $\mathbf{E}_{h}$ can be defined as

$$
\begin{aligned}
& \mathbf{s}_{h}=\left\{\mathbf{s}_{h, j}\right\}_{j=1}^{m}=\left\{\left(y_{h}(j), j\right)\right\}_{j=1}^{m}, \\
& \left|y_{h}(j)-y_{h}(j-1)\right| \leq 1, y_{h}(j)=L_{h, j}, \ldots, L_{h+1, j},
\end{aligned}
$$

where $y_{h}:[1, \ldots, m] \rightarrow\left[L_{h, j}, \ldots, L_{h+1, j}\right]$. The seam computation is done using dynamic programming in a similar way to [1]. We look for the optimal seam in the image grid $\mathbf{E}_{h}$ that minimizes the following constrained optimization problem:

$$
\mathbf{s}_{h}^{*}=\arg \min _{\mathbf{s}_{h}} \sum_{j=1}^{m} E_{\mathbf{s}_{h, j}} \text {, s.t. } L_{h, j} \leq y_{h}(j) \leq L_{h+1, j} .
$$

The first step is to traverse the image grid $\mathbf{E}_{h}$ from left to right and to compute the cumulative minimum energy $\mathbf{M}$ for all possible connected seams for each pixel location $\left(y_{h}(j), j\right)$ :

$$
\begin{aligned}
& M_{y_{h}(j), 1}=E_{y_{h}(j), 1}, \\
& M_{y_{h}(j), j}=E_{y_{h}(j), j}+\min \left\{\begin{array}{l}
M_{y_{h}(j)-1, j-1} \\
M_{y_{h}(j), j-1} \\
M_{y_{h}(j)+1, j-1}
\end{array} .\right.
\end{aligned}
$$

The minimum value of the last column in $\mathbf{M}$ will indicate the end of the minimal connected horizontal seam. In the second step we traverse the cumulative energy $\mathbf{M}$ backwards to find the path of the optimal seam. The above procedure is repeated for each image grid $\mathbf{E}_{h}$, until the whole manuscript page is processed.

\section{Parameter Selection}

The parameters of our algorithm are the number of slices $r$ for the medial seam computation, the smoothing parameter $b$ of the cubic spline filter (function csaps in MATLAB) and the standard deviation $\sigma$ of the Gaussian filter for the gradient image computation. In Table II we show the selected values for the above parameters on the applied datasets. There is no automatic way to tune these parameters, because they depend on the type of manuscript under investigation. Different parameters were used inside the collections due to the different type of pages contained in them 1 (see Section IV-A for more details on the datasets).

\footnotetext{
${ }^{1}$ The different subsets are available in the README file of our code available in our research page http://ivrg.epfl.ch/research/handwriting_ recognition/text_line_extraction
} 


\begin{tabular}{|c|c|c|c|}
\hline Collection & \multicolumn{3}{|c|}{ Parameters } \\
\hline & $r$ & $b$ & $\sigma$ \\
\hline \hline Al-Majid-A 1/2 & $4 / 4$ & $0.05 / 0.005$ & $0 / 0$ \\
Al-Majid-B & 4 & 0.005 & 0.5 \\
\hline Wadod-A spanish & 3 & 0.0005 & 0 \\
Wadod-A arabic 1/2/3 & $4 / 4 / 4$ & $0.015 / 0.005 / 0.0005$ & $0 / 0 / 0$ \\
Wadod-B & 4 & 0.001 & 0.1 \\
\hline AUB-(A,B) & 4 & 0.001 & 0 \\
\hline Thomas Jefferson & 4 & 0.001 & 0 \\
\hline Aline & 8 & 0.0003 & 3 \\
\hline
\end{tabular}

TABLE I

PARAMETER VALUES ON THE VARIOUS DATASETS.

\begin{tabular}{|c|c|c|c|}
\hline Collection & Pages & Lines & Language \\
\hline \hline Al-Majid-A/B & $96 / 7$ & $2043 / 60$ & Arabic \\
\hline Wadod-A/B & $70 / 29$ & $1229 / 211$ & Arabic-Spanish \\
\hline AUB-A/B & $40 / 13$ & $391 / 87$ & English \\
\hline Thomas Jefferson & 9 & 123 & English \\
\hline Aline & 91 & 2906 & French \\
\hline
\end{tabular}

TABLE II

DETAILS OF THE DATASETS USED IN OUR EXPERIMENTS.

The standard deviation $\sigma$ does not heavily affect the algorithm's accuracy. A positive value can be used when the manuscript images contain some amount of bleed-through noise, which can result in a more robust separating seam computation. The number of slices $r$ depends on the image resolution and text layout. A value of $r=4$ works relatively well for an average manuscript page. In the case of Aline, the value of $r=8$ is used due to the higher resolution of the image and the different layout: many text lines span only part of the page width. The smoothing parameter $b$ depends on the handwriting and script complexity. Heavy smoothing would create fewer local maxima, resulting in merged text lines. On the other hand, insufficient smoothing would create additional medial seams between text, resulting in nonrobust text lines.

\section{EXPERIMENTAL EVALUATION}

\section{A. Datasets}

We conduct experiments on the original manuscript pages of the work Aline by the Swiss-French writer Charles-Ferdinand Ramuz. We obtained it from the Bibliothèque Cantonale et Universitaire of Lausanne $(B C U)^{2}$ In order to show the applicability of our method to diverse manuscript pages, we also apply our algorithm to the dataset of [4], which is organized in four collections and contains 215 manuscript pages in Arabic (Al-Majid and Wadod), Spanish (Wadod) and English (AUB and Thomas Jefferson). Finally, we compare our algorithm with the state-of-the-art method of [4] on a smaller dataset similar to the one above. We received

\footnotetext{
${ }^{2}$ http://www.bcu-lausanne.ch/ Due to copyright reasons, the manuscript pages are not available online.
}

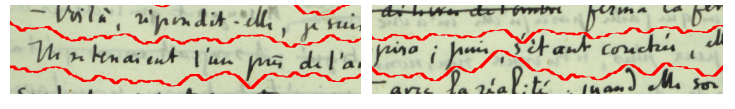

(a) Type I seams

(b) Type II seams.

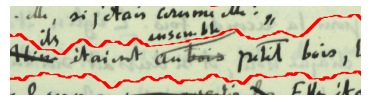

(c) Type III seam.

Fig. 4. The three seam types generated by our algorithm.

it from the authors of [4], along with their generated seams on it. The characteristics of all the datasets are shown in Table II], where the indices A,B denote the original dataset of [4] and the smaller one, respectively. We decided to compare only with the method of [4], because this is the most related algorithm to our method. In contrast to [20], it does not depend on any learning procedure that requires training and test data.

\section{B. Results}

The first evaluation of the text line extraction experiments is done manually by visually comparing the generated separating seams with the available ground truth. We also compare our algorithm with the method of [4] on the two main datasets (except Aline) of Table [I] using the automatic evaluation protocol of [4] for grayscale text line extraction. For the purposes of the manual evaluation, we distinguish between three types of seams, according to their accuracy:

1) Type I seams that pass between two consecutive text lines without cutting through any text components. Only these seams correspond to perfect text line separation.

2) Type II seams that cut through letter components or assign punctuation marks to the wrong line. These seams contain some false information about text line parts, but they are not highly inaccurate.

3) Type III seams that cut through text lines and assign word parts to the wrong line. These seams are highly inaccurate, since they contain false information about the current text line.

In Fig. 4 we show examples of the three seam types generated by our algorithm. In the last row of Table III we show results obtained by our algorithm on Aline. Most of the type II seams assign punctuation marks to the wrong line (see Fig. 4b) and only seven are the ones that cut through letter components. The manuscript of Aline contains words between text lines, which always belong to the lower one. Most of the type III seams are of this category (see Fig. 4c). Only in two cases of standard text layout did the seams assign text parts to the wrong 


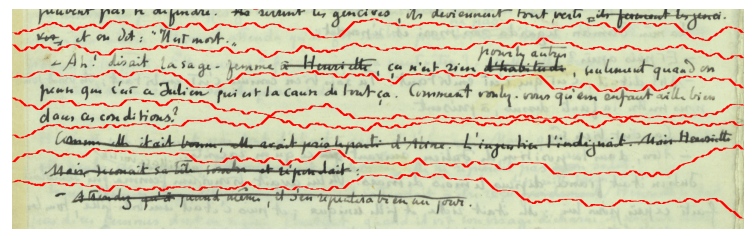

(a) Computed seams with our approach.

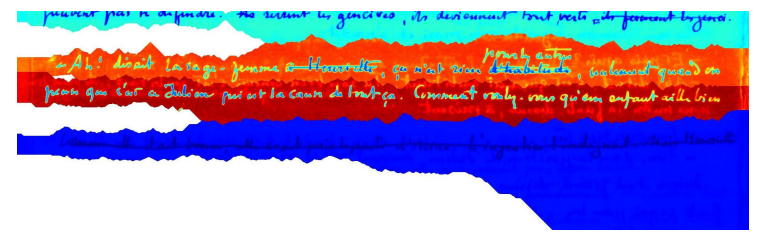

(b) Computed seams with the approach of [4].

Fig. 5. Comparison on a sample page of Aline.

\begin{tabular}{|c|c|c|c|}
\hline & \multicolumn{3}{|c|}{ Seam Type } \\
\hline Collection & I & II & III \\
\hline \hline Al-Majid-A & $93.6 \%$ & $6 \%$ & $0.4 \%$ \\
\hline Wadod-A & $64.4 \%$ & $35.3 \%$ & $0.3 \%$ \\
\hline AUB-A & $76.4 \%$ & $23.6 \%$ & $0 \%$ \\
\hline Thomas Jefferson & $54.4 \%$ & $45.6 \%$ & $0 \%$ \\
\hline Aline & $91.5 \%$ & $4.9 \%$ & $3.6 \%$ \\
\hline
\end{tabular}

TABLE III

MANUAL EVALUATION OF OUR APPROACH ON THE DATASET OF [4].

lines. In four cases, two lines are merged together and only when one of them contains just few words.

The authors of [4] gave us the output of their algorithm on a page sample from Aline. In Fig. 5 we show the generated seams from the two algorithms. The method of [4] cannot cope with partial text lines and words between lines, missing them completely (see Fig. 5b. Our algorithm, however, is able to handle such situations, which are very frequent in manuscript pages (see Fig. 5a).

The results of our algorithm on the dataset of [4] are shown in Table III As in the dataset of Aline, most of the type II seams assign punctuation marks on the wrong line. This is evident in the Arabic script of the Al-MajidA and Wadod-A collections (see Figs. 6a. 6b. In these cases, the algorithm would need to take into account language-dependent information in order to be able to correct for these failures. We observe that a fair amount of type II seams occurs in the collections of AUB-A and Thomas Jefferson. Most of the type II seams in the AUB collection assign punctuation marks to the wrong lines (see Fig. 6c). In the Thomas Jefferson collection, most of the seams cut through letter components, due to the low resolution of the images and the existence of large ascenders and descenders in the script (see in Fig. 6d the second seam). Again, as in the manuscript of Aline, we observe that only few type III seams are generated, and exclusively in pages of Arabic script. English and

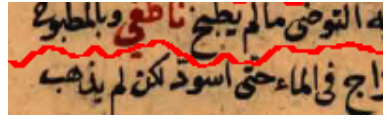

(a) Al-Majid-A

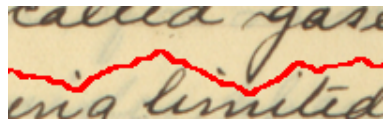

(c) AUB-A

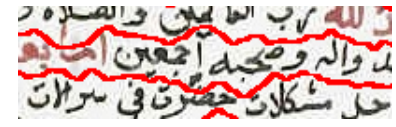

(b) Wadod-A

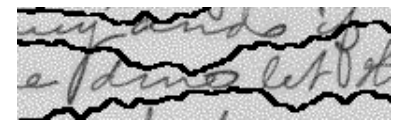

(d) Thomas Jefferson
Fig. 6. Type II seams on the dataset of [4].

\begin{tabular}{|c|c|c|c|c|c|c|}
\hline & \multicolumn{5}{|c|}{ Seam Type } \\
\hline Collection & \multicolumn{2}{|c|}{ I } & \multicolumn{2}{c|}{ II } & \multicolumn{2}{c|}{ III } \\
\hline \hline & Ours & {$[4$} & Ours & 4 & Ours & {$[4]$} \\
\hline Al-Majid-B & $\mathbf{9 8 . 2} \%$ & $69.9 \%$ & $\mathbf{1 . 8} \%$ & $26.4 \%$ & $\mathbf{0} \%$ & $3.7 \%$ \\
\hline Wadod-B & $\mathbf{7 8} \%$ & $53.9 \%$ & $\mathbf{2 1 . 5} \%$ & $45.6 \%$ & $\mathbf{0 . 5} \%$ & $\mathbf{0 . 5} \%$ \\
\hline AUB-B & $\mathbf{9 2} \%$ & $53.9 \%$ & $\mathbf{8} \%$ & $43.4 \%$ & $\mathbf{0} \%$ & $2.7 \%$ \\
\hline
\end{tabular}

TABLE IV

MANUAL COMPARISON ON THE SMALLER DATASET OF TABLEII

Spanish pages do not contain any seam of this type.

In Table $[\mathrm{IV}]$ we present a comparison between our approach and the method of [4] on the smaller dataset of Table The results of our method are similar to the ones on the larger dataset of [4]: We obtain type III seams only on Arabic script and most of the type II seams incorrectly assign punctuation marks. We consistently outperform the method of [4] on all seam types and on all data collections.

We compare both algorithms using the automatic evaluation protocol of [4] on the two datasets of Table II] The protocol uses a binary image to compute labels of the text line extraction results. The same procedure is used to label the ground truth text lines. The final accuracy is computed as the average overlap between the ground truth labels and the text line extraction labels. The resulting text line extraction accuracy is shown in Table $\mathrm{V}$. The results in the third column for the large dataset were taken directly from [4]. We observe the same behavior as in the manual evaluation of Table IV. where we consistently outperform the algorithm of [4] in all data collections. By comparing the two Tables, we can see that the type III seams mainly affect the accuracy of a text line extraction algorithm, while the type II seams only slightly influence the result. This is the case in the AUB-B collection, where our algorithm does not produce any type III seams, in contrast to [4], where their algorithm misses two text lines that do not span the whole page width.

\section{Conclusion And Future Work}

In this paper, we propose a novel text line extraction algorithm for grayscale or color scans of historical 


\begin{tabular}{|c|c|c|}
\hline & \multicolumn{2}{|c|}{ Accuracy } \\
\hline Collection & Ours & 4 \\
\hline Al-Majid-A/B & $\mathbf{9 9 . 3 0} \% / \mathbf{9 9 . 9 7} \%$ & $97.59 \% / 98.19 \%$ \\
\hline Wadod-A/B & $\mathbf{9 9 . 0 4} \% / \mathbf{9 9 . 8 7} \%$ & $98.35 \% / 97.53 \%$ \\
\hline AUB-A/B & $\mathbf{9 9 . 7 5} \% / \mathbf{9 9 . 9 7} \%$ & $98.05 \% / 96.15 \%$ \\
\hline Thomas Jefferson & $\mathbf{9 7 . 7 5} \%$ & $95.21 \%$ \\
\hline
\end{tabular}

TABLE V

COMPARISON WITH THE EVALUATION PROTOCOL OF [4].

manuscripts based on seam carving. We constrain the seam computation between two consecutive text lines using a histogram matching procedure. As a result, we are able to generate robust seams that do not cut through line components. We obtain state-of-theart results on diverse manuscript pages without any prior binarization. The code of our algorithm together with our generated seams for the dataset of [4] can be downloaded from our research page http://ivrg.epfl.ch/ research/handwriting_recognition/text_line_extraction.

The performance of our algorithm is dependent on the medial seam computation. Cases may arise where the number of local maxima is not equal for some pairs of adjacent slices. This does not pose any problem in our algorithm, because we match local maxima that agree in both directions. Only in few cases we encountered matching problems between local maxima, and these can be easily overcome with different selection of the parameters. An analytic evaluation of the medial seam computation step and its performance correlation with the separating seam computation will be investigated in future work.

\section{AKNOWLEDGMENTS}

We thank the author of [4], Abedelkadir Asi, for providing us results on a sample of Aline and on the smaller subset of their dataset collection. We also thank the Bibliothèque Cantonale et Universitaire of Lausanne for providing us the manuscript pages of Aline.

\section{REFERENCES}

[1] S. Avidan and A. Shamir, "Seam Carving for Content-Aware Image Resizing," ACM Transactions on Graphics, vol. 26, no. 3, p. 10, 2007.

[2] M. Liwicki, E. Indermuhle, and H. Bunke, "On-line handwritten text line detection using dynamic programming," in International Conference on Document Analysis and Recognition, vol. 1, 2007, pp. 447-451.

[3] J. Sauvola and M. Pietikäinen, "Adaptive document image binarization," Pattern Recognition, vol. 33, no. 2, pp. 225-236, 2000.

[4] R. Saabni, A. Asi, and J. El-Sana, "Text line extraction for historical document images," Pattern Recognition Letters, vol. 35, no. 0, pp. 23-33, 2014.

[5] U. Marti and H. Bunke, "Using a Statistical Language Model to Improve the Performance of an HMM-based Cursive Handwriting Recognition System," International Journal of Pattern Recognition and Artificial Intelligence, vol. 15, no. 01, pp. 65-90, 2001.
[6] M. Bulacu, R. van Koert, L. Schomaker, and T. van der Zant, "Layout Analysis of Handwritten Historical Documents for Searching the Archive of the Cabinet of the Dutch Queen," in International Conference on Document Analysis and Recognition, 2007, pp. 357-361.

[7] G. Louloudis, B. Gatos, I. Pratikakis, and C. Halatsis, "Text line detection in handwritten documents," Pattern Recognition, vol. 41, no. 12, pp. 3758-3772, dec 2008.

[8] L. Likforman-Sulem, A. Hanimyan, and C. Faure, "A Hough based algorithm for extracting text lines in handwritten documents," in International Conference on Document Analysis and Recognition, vol. 2, 1995, pp. 774-777.

[9] K. Wong and F. Wahl, "Document analysis system," IBM Journal of Research and Development, vol. 26, pp. 647-656, 1982.

[10] Z. Shi and V. Govindaraju, "Line Separation for Complex Document Images Using Fuzzy Runlength," in International Workshop on Document Image Analysis for Libraries, 2004, pp. 306-312.

[11] N. Nikolaou, M. Makridis, B. Gatos, N. Stamatopoulos, and N. Papamarkos, "Segmentation of historical machine-printed documents using Adaptive Run Length Smoothing and skeleton segmentation paths," Image and Vision Computing, vol. 28, no. 4, pp. 590-604, April 2010.

[12] A. Fischer, M. Wuthrich, M. Liwicki, V. Frinken, H. Bunke, G. Viehhauser, and M. Stolz, "Automatic Transcription of Handwritten Medieval Documents," in International Conference on Virtual Systems and Multimedia, 2009, pp. 137-142.

[13] A. Fischer, E. Indermühle, H. Bunke, G. Viehhauser, and M. Stolz, "Ground Truth Creation for Handwriting Recognition in Historical Documents," in IAPR International Workshop on Document Analysis Systems, 2010, pp. 3-10.

[14] S. Bukhari, F. Shafait, and T. Breuel, "Script-Independent Handwritten Textlines Segmentation Using Active Contours," in International Conference on Document Analysis and Recognition, 2009, pp. 446-450.

[15] S. Bukhari, F. Shafait, and T. Breuel, "Text-Line Extraction Using a Convolution of Isotropic Gaussian Filter with a Set of Line Filters," in International Conference on Document Analysis and Recognition, 2011, pp. 579-583.

[16] A. Nicolaou and B. Gatos, "Handwritten Text Line Segmentation by Shredding Text into its Lines," in International Conference on Document Analysis and Recognition, 2009, pp. 626-630.

[17] N. Ouwayed and A. Belaï, "A general approach for multioriented text line extraction of handwritten documents," International Journal on Document Analysis and Recognition, vol. 15, no. 4 , pp. 297-314, 2012.

[18] A. Garz, A. Fischer, R. Sablatnig, and H. Bunke, "BinarizationFree Text Line Segmentation for Historical Documents Based on Interest Point Clustering," in IAPR International Workshop on Document Analysis Systems, 2012, pp. 95-99.

[19] A. Garz, A. Fischer, H. Bunke, and R. Ingold, "A BinarizationFree Clustering Approach to Segment Curved Text Lines in Historical Manuscripts," in International Conference on Document Analysis and Recognition, August 2013, pp. 1290-1294.

[20] M. Baechler, M. Liwicki, and R. Ingold, "Text Line Extraction using DMLP Classifiers for Historical Manuscripts," in International Conference on Document Analysis and Recognition, August 2013, pp. 1029-1033.

[21] A. Fischer, H. Bunke, N. Naji, J. Savoy, M. Baechler, and R. Ingold, "The HisDoc Project. Automatic Analysis, Recognition, and Retrieval of Handwritten Historical Documents for Digital Libraries," in InterNational and InterDisciplinary Aspects of Scholarly Editing, February 2012.

[22] P. Toivanen, "New geodesic distance transforms for gray-scale images," Pattern Recognition Letters, vol. 17, no. 5, pp. 437450, 1996. 